\title{
Chromatin accessibility and regulatory vocabulary across indicine cattle tissues
}

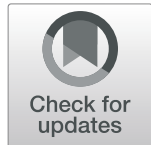

\author{
Pâmela A. Alexandre ${ }^{1 * \dagger}$ (D), Marina Naval-Sánchez ${ }^{1,2+}$, Moira Menzies ${ }^{1}$, Loan T. Nguyen³ ${ }^{3}$ Laercio R. Porto-Neto ${ }^{1}$,
} Marina R. S. Fortes ${ }^{4}$ and Antonio Reverter ${ }^{1}$

\author{
* Correspondence: pamela. \\ alexandre@csiro.au \\ ${ }^{\dagger}$ Pâmela A. Alexandre and Marina \\ Naval-Sánchez contributed equally \\ to this work. \\ ${ }^{1}$ CSIRO Agriculture \& Food, 306 \\ Carmody Rd., QLD 4067 Brisbane, \\ Australia \\ Full list of author information is \\ available at the end of the article
}

\begin{abstract}
Background: Spatiotemporal changes in the chromatin accessibility landscape are essential to cell differentiation, development, health, and disease. The quest of identifying regulatory elements in open chromatin regions across different tissues and developmental stages is led by large international collaborative efforts mostly focusing on model organisms, such as ENCODE. Recently, the Functional Annotation of Animal Genomes (FAANG) has been established to unravel the regulatory elements in non-model organisms, including cattle. Now, we can transition from prediction to validation by experimentally identifying the regulatory elements in tropical indicine cattle. The identification of regulatory elements, their annotation and comparison with the taurine counterpart, holds high promise to link regulatory regions to adaptability traits and improve animal productivity and welfare.
\end{abstract}

Results: We generate open chromatin profiles for liver, muscle, and hypothalamus of indicine cattle through ATAC-seq. Using robust methods for motif discovery, motif enrichment and transcription factor binding sites, we identify potential master regulators of the epigenomic profile in these three tissues, namely HNF4, MEF2, and SOX factors, respectively. Integration with transcriptomic data allows us to confirm some of their target genes. Finally, by comparing our results with Bos taurus data we identify potential indicine-specific open chromatin regions and overlaps with indicine selective sweeps.

Conclusions: Our findings provide insights into the identification and analysis of regulatory elements in non-model organisms, the evolution of regulatory elements within two cattle subspecies as well as having an immediate impact on the animal genetics community in particular for a relevant productive species such as tropical cattle.

Keywords: Bos indicus, ATAC-seq, Motif discovery, Open chromatin region

\section{Background}

Chromatin is a complex of DNA and proteins (nucleosomes) found in the nucleus of eukaryotic cells. The non-uniform topological organization of nucleosomes across the genome, as well as their post-translational modifications, reflects a dynamic process that controls chromatin accessibility, switching between transcriptionally active

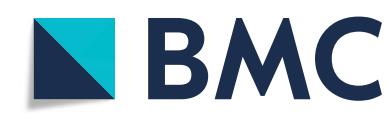

(c) The Author(s). 2021 Open Access This article is licensed under a Creative Commons Attribution 4.0 International License, which permits use, sharing, adaptation, distribution and reproduction in any medium or format, as long as you give appropriate credit to the original author(s) and the source, provide a link to the Creative Commons licence, and indicate if changes were made. The images or other third party material in this article are included in the article's Creative Commons licence, unless indicated otherwise in a credit line to the material. If material is not included in the article's Creative Commons licence and your intended use is not permitted by statutory regulation or exceeds the permitted use, you will need to obtain permission directly from the copyright holder. To view a copy of this licence, visit http://creativecommons.org/licenses/by/4.0/. The Creative Commons Public Domain Dedication waiver (http://creativecommons.org/publicdomain/zero/1.0/) applies to the data made available in this article, unless otherwise stated in a credit line to the data. 
euchromatin and inactive heterochromatin [1]. The landscape of chromatin accessibility regulates the degree to which nuclear macromolecules can bind the double helix, thus affecting transcription, DNA repair, replication, and recombination [2]. Nucleosomes are known to be depleted at regulatory loci, including enhancers, insulators, and transcribed gene bodies, making binding sites available to transcription factors (TFs) and the transcription machinery [3]. These epigenetic changes are instrumental to cell differentiation, environmental signaling, and disease development. The quest of identifying regulatory elements in open chromatin regions across different tissues and developmental stages is led by large international consortia mostly focussing on model organisms, such as the Encyclopedia of DNA Elements (ENCODE) in humans [4, 5], the mouseENCODE for mouse [6] and the modENCODE for fruitfly and C. elegans [7, 8].

Recently, the Functional Annotation of Animal Genomes (FAANG [9]) and its counterpart, Functional Annotation of All Salmonid Genomes (FAASG [10]), have been established with the aim to unravel the regulatory elements in non-model organisms, including chicken, pig, cattle, ovine, and aquaculture species. In this context, our group has contributed the first draft of cattle and sheep functional regulatory regions based on the identification of orthologous regulatory regions $[11,12]$ from human and mouse $[4,6,13]$. However, particularly in cattle, the possibility of investigating chromatin accessibility sheds light on the expected differences between the two subspecies, Bos taurus indicus and Bos taurus taurus [14, 15]. Indicine (or zebu) breeds (B. indicus) are highly adapted to tropical environments, including resistance to disease and parasites, heat stress, and severe drought conditions. Considering more than half of livestock heads are found in tropical and subtropical environments [16], understanding and selecting animals for adaptability traits is of high economic and welfare relevance. The functional genomic basis of climatic adaptation in beef cattle is not well understood, and resolving tissue-specific deployment of regulatory activity directed by small sequences is paramount.

In the quest of detecting chromatin accessibility, the Assay of Transposase Accessible Chromatin sequencing (ATAC-seq) has become increasingly popular [1]. The libraries for ATAC-seq are constructed by incorporating a hyperactive Tn5 transposase that simultaneously cuts open chromatin on both ends, leaving a 9 bp staggered nick. Then, high-throughput sequencing adapters are ligated to these regions [17]. PCR is used for library construction, followed by paired-end next-generation sequencing. This simple and fast protocol, paired with its high sensitivity and low requirement for starting cell number, are the reasons for the popularity of this assay [1]. Recently, ATAC-seq data has been used to annotate and compare domesticated farm animals, namely bovine ( $B$. taurus), chicken, goat, and pig $[18,19]$. Here, we generated open chromatin profiles for three tissues (liver, muscle, and hypothalamus) of tropical cattle (B. t. indicus) through ATAC-seq. The liver was chosen for being a central organ of metabolism, including bilirubin, bile acids, carbohydrates, lipids, xenobiotics, protein synthesis, and immunity [20]. Similarly, the hypothalamus is a representative of the neuroendocrine system involved in the regulation of several body processes, such as stress reaction, digestion, immunity, behavior, sexual behavior, and energy storage and expenditure. Finally, the skeletal muscle was chosen for being the ultimate focus for beef cattle production.

Using several bioinformatics approaches, such as motif discovery, as well as publicly available datasets, we aimed to functionally characterize regulatory elements in indicine 
tissues as well as their underlying regulatory code. That is the combination of transcription factor binding sites (TFBS) that govern the spatiotemporal regulatory activity and gene expression. This improved knowledge of regulatory annotation sheds high promise in linking sequence to phenotype and posing new questions on our current understanding of productive traits of agricultural relevance.

\section{Results}

\section{Regulatory landscapes across three tissues in tropical cattle}

To annotate regulatory elements in tropical cattle, we generated ATAC-seq data from liver, hypothalamus, and muscle of three post-puberty Brahman heifers [21-23]. After quality control, samples resulted in an average of 82,988,361 uniquely mapped reads (Additional file 1), in agreement with the quality standards determined by ENCODE ATAC-seq pipeline [24].

Open chromatin regions were identified through consensus peaks across biological replicates in each tissue, resulting in 78,528 peaks for hypothalamus, 40,104 peaks for muscle, and 22,291 peaks for liver (Additional files 2-4); covering 2.41\%, $0.98 \%$, and $0.52 \%$ of the genome, respectively (Additional file 5 ). The average peak length was 836 bp, $667 \mathrm{bp}$, and $635 \mathrm{bp}$ for hypothalamus, muscle, and liver, respectively (Table 1).

A signal of good quality in ATAC-seq data is to present enrichment for transcription start sites (TSS), which can be seen in Fig. 1A. However, open chromatin regions can be mapped into different functional categories, including gene bodies, promoters, and distal regulatory elements (Fig. 1B). Most of our called peaks fall within distal intergenic (41$55 \%)$, followed by promoter regions (18-50\%). A small percentage of peaks fall within exons $(0.8-3 \%)$ and untranslated regions (UTR, $<1 \%)$. Although peaks are assigned to the most representative genomic feature to allow for easy comparison across tissues, often the same peak can span multiple features, which was captured in Additional file 6. It is noteworthy that samples with a lower number of peaks present a higher percentage of peaks within Promoter/TSS regions. This behavior is also observed for the distribution of peaks in terms of distance to the TSS of the nearest gene (Fig. 1C).

\section{Genome-wide differences in chromatin accessibility profiles across tissues}

The identified peaks were compared between tissues and tissue-specific peaks were defined. Conversely, overlapping regions in all three tissues were considered constitutive. We were able to identify $2213,11,439$, and 53,289 tissue-specific peaks for liver, muscle,

Table 1 Peak calling metrics

\begin{tabular}{|c|c|c|c|c|c|}
\hline & $\begin{array}{l}\text { Total peaks } \\
\text { identified }\end{array}$ & $\begin{array}{l}\text { Consensus peaks } \\
(P<0.01)\end{array}$ & $\begin{array}{l}\text { Average peak } \\
\text { length (bp) }\end{array}$ & $\begin{array}{l}\text { Peaks on } \\
\text { chr1-29 } \\
\text { and } X\end{array}$ & $\begin{array}{l}\text { Proportion of } \\
\text { peaks near TSS } \\
( \pm 3 \mathrm{~Kb}, \%)\end{array}$ \\
\hline Hypothalamus & $212,636,473$ & 78,528 & 836 & 71,028 & 18.07 \\
\hline Liver & $285,783,943$ & 22,291 & 635 & 12,063 & 50.54 \\
\hline Muscle & $248,240,326$ & 40,104 & 667 & 30,483 & 30.95 \\
\hline Hypothalamus-specific & - & 53,289 & 630 & 53,103 & 9.08 \\
\hline Liver-specific & - & 2213 & 361 & 938 & 9.49 \\
\hline Muscle-specific & - & 11,439 & 474 & 10,976 & 7.56 \\
\hline Constitutive & - & 11,983 & 578 & 9803 & 59.37 \\
\hline
\end{tabular}




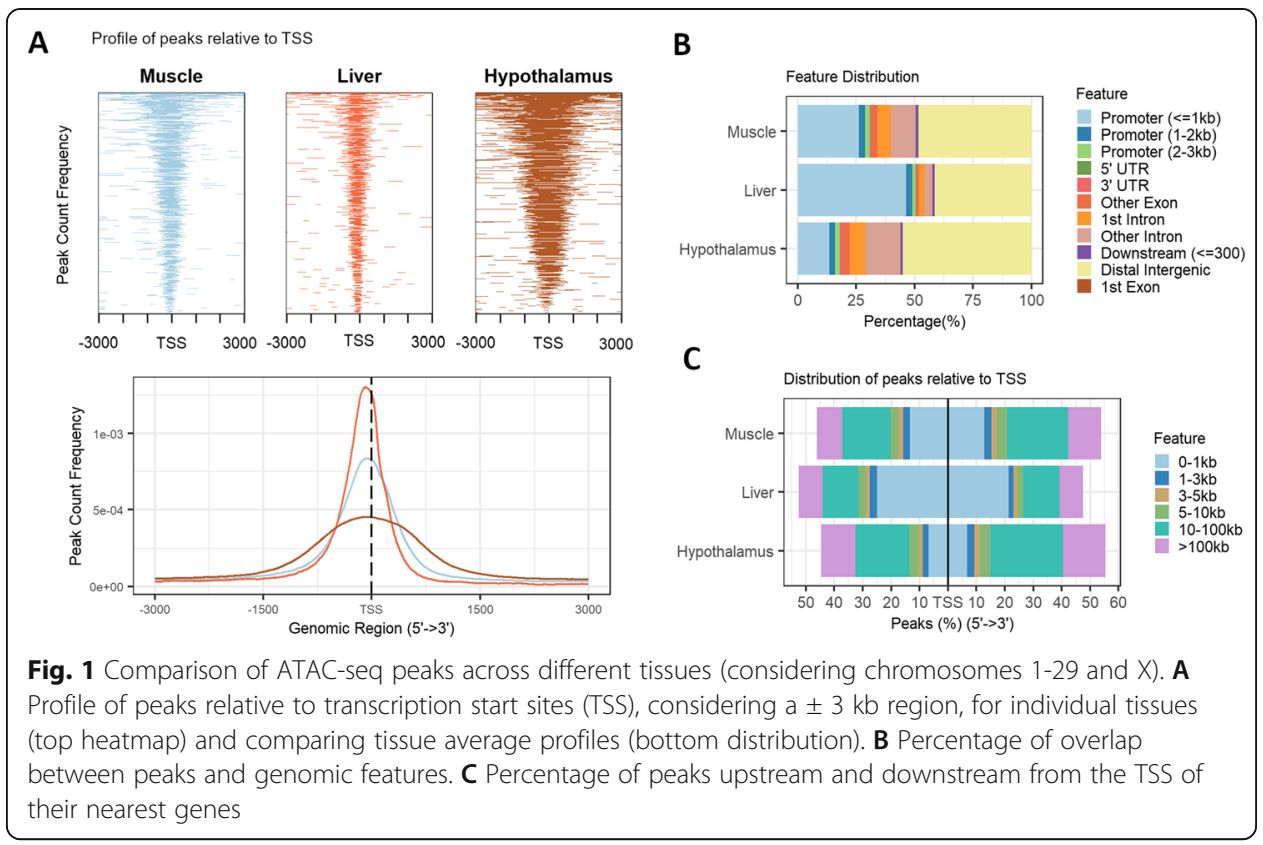

and hypothalamus, respectively, and 11,983 constitutive regions (Additional files 2-4, 7). For the four subsets, an enrichment around TSS can still be seen (Fig. 2A). However, while more than half of constitutive regions lie in promoter regions and gene bodies, most tissue-specific peaks fall into intergenic and intronic regions (Fig. 2B).

Assigning peaks to genomic features invites relating those peaks to the nearest annotated gene and, therefore, identifying key biological functions associated with open chromatin regions (Fig. 2C, Additional file 8). Muscle-specific peaks were associated mostly with terms related to muscle tissue development (Padj $=2.13 \mathrm{E}-06)$ and muscle system process $($ Padj $=1.12 \mathrm{E}-05)$. Hypothalamus presented enrichment for terms related to cell communication such as cell surface receptor signaling pathway (Padj = $0.04)$ and for nervous system development (Padj $=0.04)$. Liver only presented enrichment for positive chemotaxis (Padj $=1.10 \mathrm{E}-06)$ which might be related to the movement of immune-competent cells characteristics of this tissue. Finally, constitutive regions were mostly related to RNA processing (Padj $=5.93 \mathrm{E}-10)$, translation (Padj $=$ $1.54 \mathrm{E}-06)$, and protein catabolic process (Padj $=1.14 \mathrm{E}-06)$.

\section{Motif discovery unravels master tissue-specific regulators}

The 2213 liver-specific ATAC-seq peaks were converted into 546 human genome regions which were enriched for master regulators of liver and hepatocyte differentiation, namely hepatocyte nuclear factors HNF4A/G (normalized enrichment score-NES = 12.47), and HNF1A/B (NES = 10.59) (Fig. 3A, Additional file 9). The enrichment analysis of our liver-specific regions against a public TF ChIP-seq bound regions database in human cell lines from ENCODE confirmed the experimental binding of HNF4G on human HepG2 cells as the most enriched track (ENCFF001UGI, NES $=7.39$ ), followed by RXRA (ENCFF001UHJ, NES $=6.81$ ) and HNF4A (ENCFF001UGH, NES = 6.79; ENCFF001UGG, NES = 6.78). Using the same methodology, we confirmed our liverspecific regions were enriched for open chromatin in hepatocyte cell lines from 

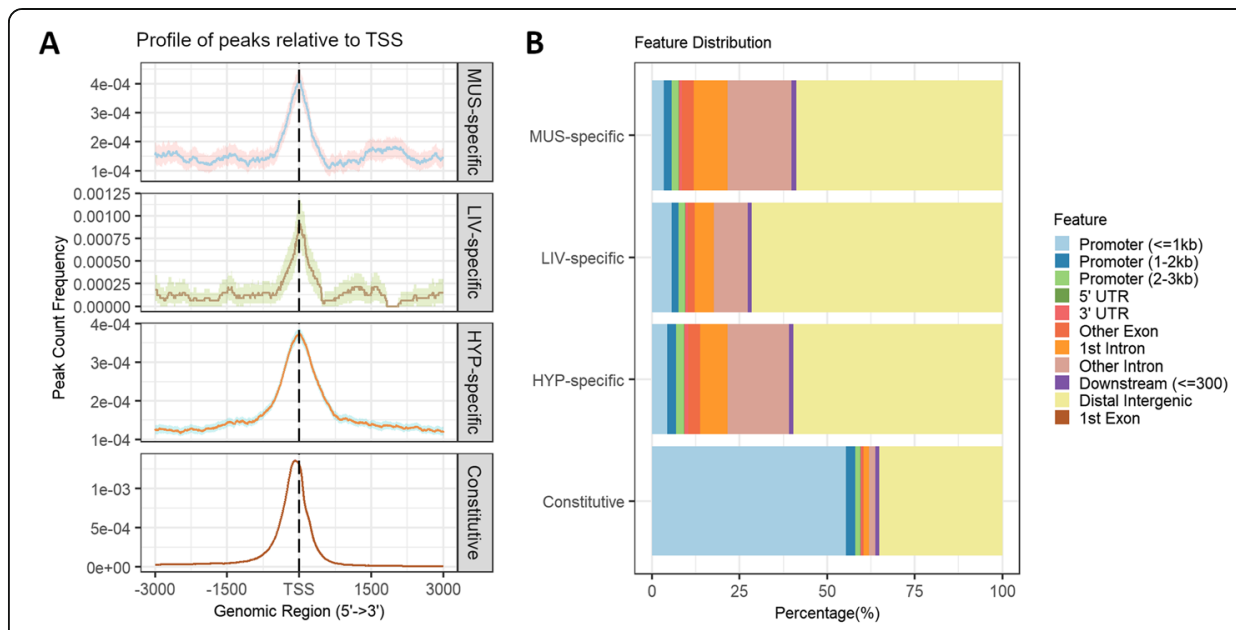

C

GO Enrichment Analysis

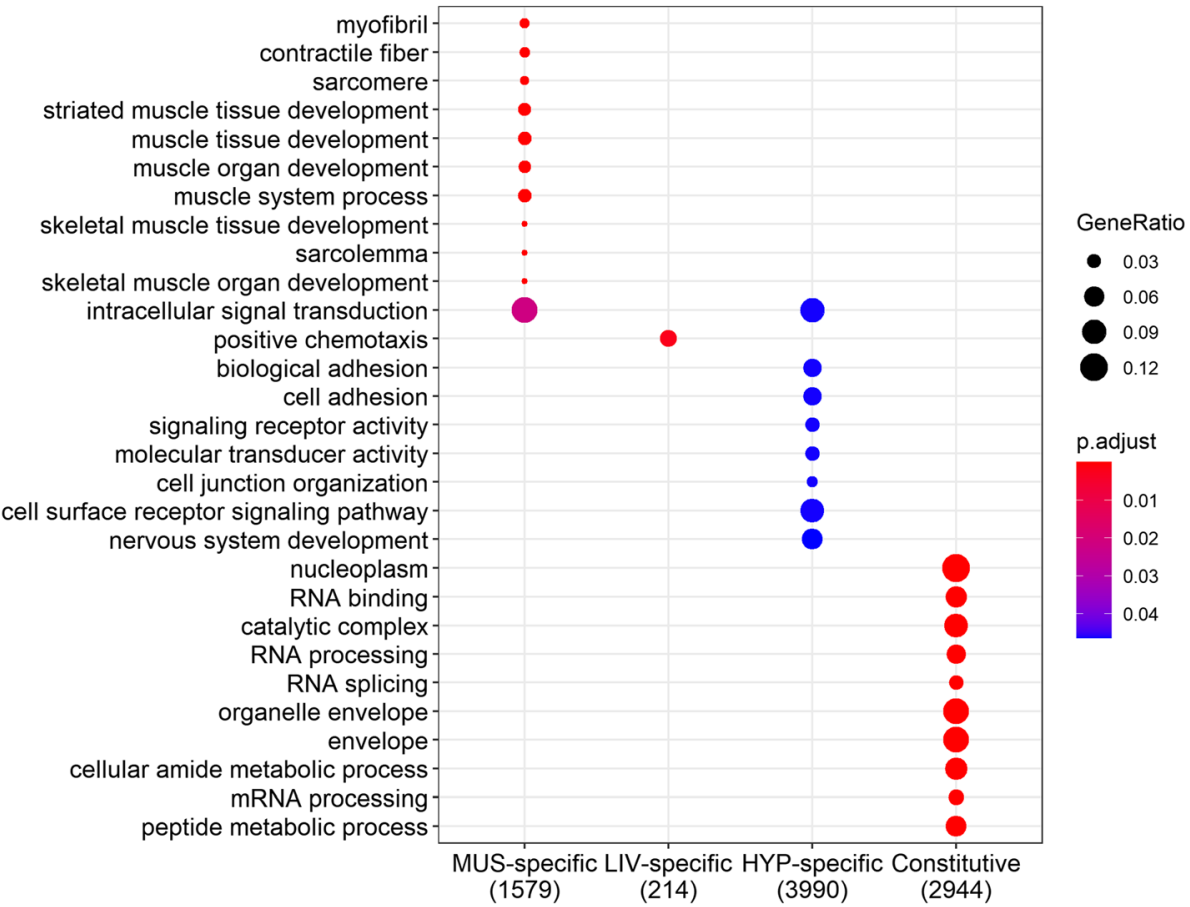

Fig. 2 Comparison between tissue-specific (TS) peaks and constitutive regions for muscle (MUS), liver (LIV), and hypothalamus (HYP). A Profile of peaks relative to transcription start sites (TSS) considering a $\pm 3 \mathrm{~kb}$ region-confidence intervals were estimated by bootstrap method (500 iterations) and is shown as the shading that follows each curve. B Percentage of overlap between peaks and genomic features. $\mathbf{C}$ Functional enrichment of top 10 gene ontology $(\mathrm{GO})$ terms for genes associated to peaks

ENCODE, namely H3K27ac in HEpG2 Hepatocellular carcinoma cell line (NES = 8.55) and FAIRE-seq on HepG2 (ENCFF001UYN, NES $=8.55$ ), thus strongly indicating our regions are functionally active in hepatocytes. When we converted the predicted target regions of HNF4 back to cattle coordinates and compared them with our open chromatin regions in liver, we were able to annotate 27 possible binding sites, with scores (log likelihood ratios) varying from 0.03 to 12.5 (Additional file 10). No exact score threshold exists and therefore, we reported scores for all identified target regions. Nevertheless, the higher the score the better. 


\begin{tabular}{|c|c|c|c|c|}
\hline $\begin{array}{l}\text { Cluster Rank } \\
\text { (PWM rank) }\end{array}$ & Associated TF & NES & \#Targets & Logo \\
\hline $1(1)$ & HNF4A/G & 12.47 & $\begin{array}{c}66 \\
\text { (62 genes) }\end{array}$ & 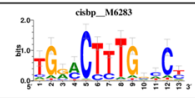 \\
\hline $2(2)$ & NR1H3 & 11.42 & $\begin{array}{c}69 \\
\text { (63 genes) }\end{array}$ & 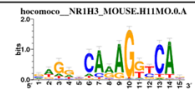 \\
\hline $3(4)$ & HNF1A/B & 10.59 & $\begin{array}{c}35 \\
\text { (35 genes) }\end{array}$ & : \\
\hline $4(32)$ & HDAC2 & 8.43 & $\begin{array}{c}48 \\
\text { (46 genes) }\end{array}$ & 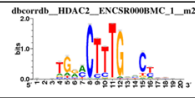 \\
\hline $5(35)$ & RXRA & 8.14 & $\begin{array}{c}51 \\
\text { (51 genes) }\end{array}$ & \\
\hline
\end{tabular}

B

\begin{tabular}{|cccc|}
\hline $\begin{array}{c}\text { Cluster Rank } \\
\text { (PWM rank) }\end{array}$ & Associated TF & NES & \#Targets
\end{tabular}

C

\begin{tabular}{|ccccc|}
\hline $\begin{array}{c}\text { Cluster Rank } \\
\text { (PWM rank) }\end{array}$ & Associated TF & NES & \#Targets & Logo \\
\hline $1(1)$ & SOX8/9/10 & 5.48 & $\begin{array}{c}3,165 \\
(2,241 \text { genes) }\end{array}$ \\
\hline $2(9)$ & ZCCHC14 & 3.86 & $\begin{array}{c}2,865 \\
(2,164 \text { genes })\end{array}$ \\
\hline $3(11)$ & PDLIM5 & 3.64 & $\begin{array}{c}2,923 \\
(2,181 \text { genes })\end{array}$ \\
\hline $4(12)$ & SOX3 & 3.62 & $\begin{array}{c}2,893 \\
(2,082 \text { genes })\end{array}$ \\
\hline $5(13)$ & SOX6 & 3.59 & $\begin{array}{c}2,240 \\
(1,664 \text { genes })\end{array}$
\end{tabular}

Fig. 3 Top 5 iRegulon motif discovery results on liver-specific (A), muscle-specific (B), and hypothalamusspecific (C) open chromatin regions 
Finally, we validated the predicted targets of HNF4 by testing their co-expression based on RNA-seq data. From the 62 predicted HNF4 targets, 52 were considered expressed in our liver data. In addition, except for HNF1B, all top TF enriched in liver presented gene expression and were included in the co-expression analysis. From 52 expressed targets, 20 (38\%) presented significant co-expression with HNF4 (Fig. 4, Additional file 11). Also, HNF1A was the only top TF co-expressed with HNF4.

The 11,439 muscle-specific ATAC-seq peaks were converted into 10,067 human genome regions which were enriched for a family of master regulators of muscle differentiation (Fig. 3B, Additional file 12), namely myocyte enhancer factor-2 (MEF2, NES = 11.71). To validate our predictions, we looked at the enrichment for ENCODE ChIP-seq experiments which resulted in skeletal muscle cell lines both in male (E107-H3K4me1, $\mathrm{NES}=8.44$; E107-H3K4me1-broadpeak, NES = 5.52) and female (E108-H3K27ac, NES = 7.50; E108-H3K4me1, NES = 7.04) and FAIRE-seq on the skeletal myoblasts cell line LHCN-M2 (ENCFF001WPB, NES $=5.23$ ) as the most enriched tracks. These results confirm our muscle-specific regions are indeed functionally active in muscle cells. By converting the predicted target regions of MEF2 back to cattle coordinates and comparing them with our open chromatin regions in muscle, we identified 667 possible binding sites, with scores varying from 3.25E-03 to 23.4 (Additional file 13).

The 53,289 hypothalamus-specific ATAC-seq peaks were converted into 48,067 human genome regions which were enriched for an important family of transcription factors for neuronal development (Fig. 3C, Additional file 14), namely SRY-related HMG box genes (SOX, NES $=5.48$ ). The SOX family can regulate several different aspects of development in general, which explains the enrichment for FAIRE-seq for Foreskin Melanocyte Primary Cells as the top enriched track (E059-DNase.hotspot.all.peaks-narrowpeak NES $=6.98$ ) and DNase-seq on human iPS (ENCFF001SPB, NES = 5.96) as the third. Nevertheless, among the top 10 enriched tracks are ENCODE ChIP-seq results for Brain Inferior Temporal Lobe (E072-H3K27ac, NES = 5.97; E072-H3K4me1, NES $=5.32$ ), Brain Substantia Nigra (E074-H3K27ac, NES = 5.80; E074-H3K4me1,

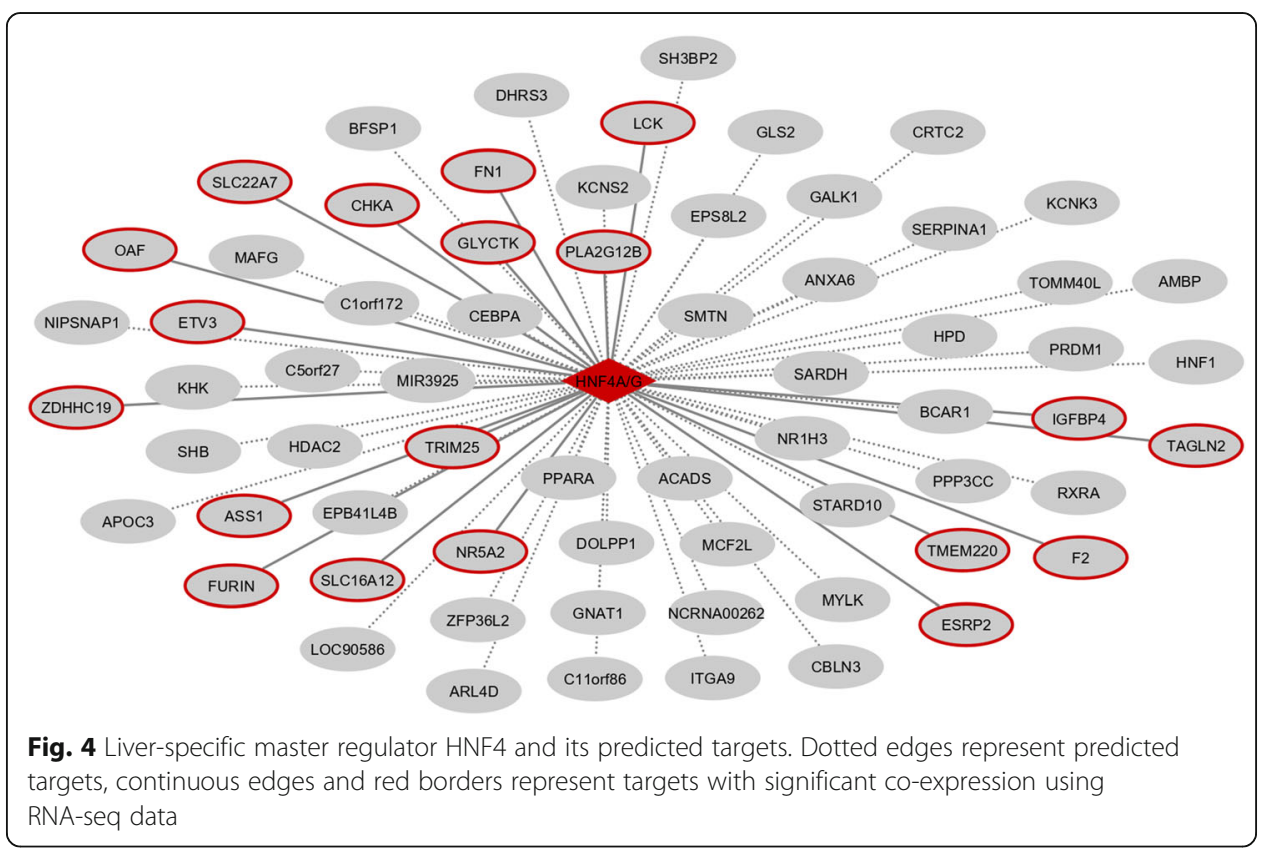


NES $=5.62)$, and Brain Hippocampus Middle (E071-H3K4me1, NES = 5.41). SOX targets coordinate when compared with our cattle open chromatin regions in hypothalamus, represented 2166 possible binding sites, with scores varying from 4.05E-06 to 18.8 (Additional file 15).

Considering RNA-seq data in hypothalamus, from the 2241 predicted SOX targets, 1632 presented gene expression, including the top TFs with the only exception of SOX3. From the expressed targets included in the co-expression analysis, 360 (22\%) presented significant results (Additional file 16, Additional file 17). Among those, SOX8/9/10 were co-expressed with other members of SOX family, namely SOX1, SOX2, SOX5, SOX6, SOX13, and SOX21.

Although publicly available data on open chromatin regions of $B$. taurus were generated using different sex and methods [19], it provides us with a unique opportunity to identify possible indicine-specific regulatory regions. While we identified 78,528 peaks for hypothalamus, 40,104 peaks for muscle and 22,291 peaks for liver in B. indicus, the correspondent numbers in B. taurus data were $20,045,77,378$, and 58,853 , respectively. Indicine-specific peaks falling on chr1-29 and X totalized 54,971 for hypothalamus, 4216 for muscle, and 2217 for liver (Additional file 18). Clearly, the higher number of peaks in hypothalamus identified in our study in comparison with the B. taurus data resulted in an inflation of indicine-specific peaks in that tissue, and therefore, the results need to be evaluated with caution. Apart from hypothalamus, indicine-specific peaks seem to be depleted from TSS regions (Fig. 5A) and concentrated on distal intergenic regions (Fig. 5B). Peaks in intergenic regions represented 57\%, 63\%, and $82 \%$ of indicine-specific peaks in hypothalamus, muscle, and liver, respectively, followed by $24 \%, 17 \%$, and $10 \%$ in introns. Indicine-specific peaks in promoter regions only accounted for $12 \%, 12 \%$, and $7 \%$, respectively.

When we compared indicine-specific peaks with previously identified selective sweeps for indicus cattle [25], we found 3, 6, and 31 peaks with an overlap in liver, muscle, and hypothalamus, respectively (Additional file 19). Most of the overlapping peaks were located on chromosome 5 and, in all tissues, they felt in distal intergenic regions with the closest genes being MIR763 and LEMD3 (Fig. 5C). In addition, in hypothalamus the peaks on chromosome 5 also were distal to HELB and IRAK3, and in the promoter region of TMBIM4 and LEMD3. Peaks in other chromosomes only appeared in hypothalamus including chr4, chr6, chr8, chr12, chr18, and chr22. Among those, we can highlight the ones overlapping promoter regions of genes MEPE, FRY, and ZNF19.

\section{Discussion}

The vast non-coding portion of the bovine genome, the one that regulates epigenetics changes, responds to environmental stimuli, and determines cell and tissue activity, is only starting to be characterized $[12,18,19]$. This non-coding genome is the key to understanding the expected differences between $B$. indicus and B. taurus and uncover the genetic basis of adaptability of indicine cattle to tropical and subtropical environments. Here, we used ATAC-seq data from indicine tissues that are key to adaptability and beef production (liver, muscle, and hypothalamus) not only to identify regulatory elements but to annotate their possible biding sites and targets in each tissue. We were able to identify HNF4 as a key regulator in liver, MEF2 in muscle and SOX in hypothalamus, and support those results based on gene co-expression and publicly available 


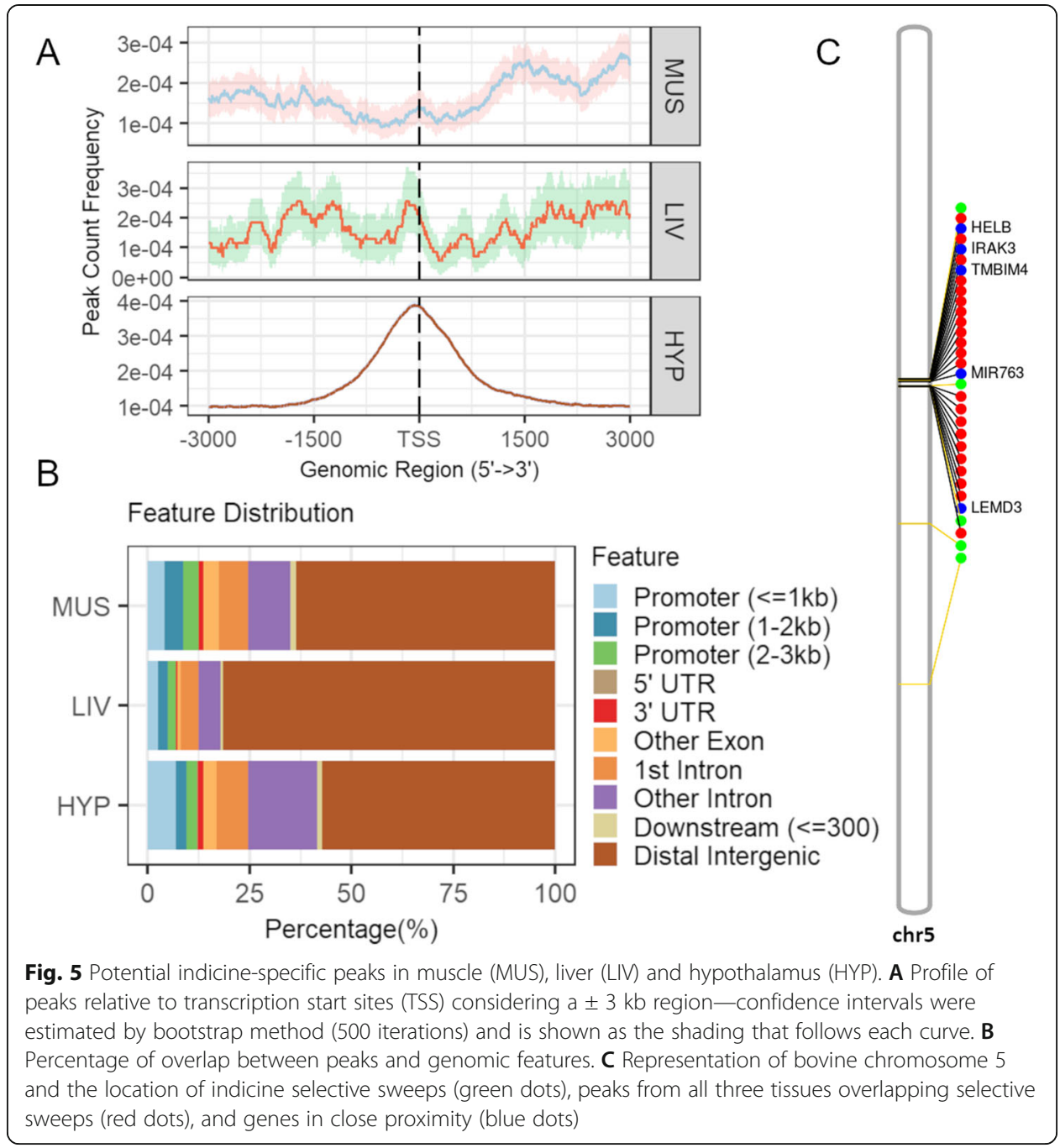

ChIP-seq and FAIRE-seq data. In addition, we compared B. indicus and B. taurus data and identified potential indicine-specific open chromatin regions, which mostly correspond to distal regulatory elements.

The peaks detected in the ATAC-seq data are expected to correspond to regulatory regions harboring functional combinations of regulatory elements, which dictate their spatiotemporal function [26]. Unravelling this regulation is the key to understanding the molecular mechanisms that control gene expression. For all our three tissues, most peaks fall within intergenic and promoter regions. This distribution of genomic features is in accordance with literature in human, mouse, and livestock species [18, 19], and correspond to two main types of regulatory elements involved in transcriptional regulation: promoters and enhancers. While promoters are located up to a few kilobases from a TSS, enhancers can be located long distances upstream or downstream of a target gene [27].

The proportion of peaks falling in promoters and intergenic regions changes when we look at tissue-specific peaks and constitutive regions. Although we can still see an enrichment around TSS for all subsets, the percentage of tissue-specific peaks within promoter regions drop dramatically (see Table 1), while for constitutive regions this 
percentage increases. This behavior suggests tissue-specific functions are more finely regulated by long-range regulatory elements, such as enhancers, silencers, and insulators. Indeed, it has been reported before that major tissue differences are due to changes in distal elements [4, 5, 28]. Conversely, constitutive regions represent housekeeping functions, and therefore, promoter regions are expected to be of open chromatin. In terms of transcriptional regulation, this distinction between constitutive/housekeeping vs. regulated/regulatory/developmental genes has proven to represent a real biological distinction rather than a human-defined classification [29].

Liver presented the smallest number of identified peaks and, consequently, of tissuespecific peaks. It also presented the highest proportion of tissue-specific peaks located in distal intergenic regions. This could be the reason why only one gene ontology term was enriched when considering the peaks nearest genes-positive chemotaxis. Chemotaxis refers to the directional migration of cells in response to a chemical stimulus, which is part of normal function and health in humans, such as immune system cells fighting injuries and infections, and tissue regeneration [30]. Liver is a frontline immune organ, responsible for detecting and clearing bacteria, viruses, and macromolecules from the blood, and is populated with several immune-centric cell types, including Kupfer cells, T cells, and NK cells [31]. In a healthy liver, metabolic functions and tissue remodeling are both requisites to maintain homeostasis [32]. In this context, hepatocyte nuclear factors (HNF) are transcription factors expressed predominately in liver. They work synergistically to raise transcriptional levels of distinct sets of hepatocyte-specific genes responsible for tissue development and metabolic homeostasis, among other functions [33, 34]. In our study, HNF4 was identified as the key regulator of liver-specific expression, but HNF1 also appear among the top TFs and both present co-expression in our RNA-seq data. Although HNF4 isoforms comprise two genes (HNF4A and HNF4G), a comparison of the DNA-binding domain of humans showed high homology between them, suggesting both genes may have similar functions in the transcriptional regulation of hepatic genes [35]. Importantly, in beef cattle, HNF4G has already been pointed as a key regulator when considering 29 traits (including meat quality, conformation, development, and metabolism) based on a markerderived gene network [36].

Genes associated with muscle-specific peaks were enriched mostly with muscle cell development and MEF2 was pointed as a master regulator of muscle-specific open chromatin regions. The myocyte enhancer factor 2 family of transcription factors is comprised of variants $\mathrm{A}, \mathrm{B}, \mathrm{C}$, and $\mathrm{D}$ with highly conserved protein domains across the MEF2 family [37]. In our study, MEF2A appear as the top enriched feature, and although it is expressed in various tissues/organs and plays crucial roles in multiple biological processes, it is widely present in muscle cells and involved in the development and differentiation of vertebrate skeletal, cardiac, and smooth muscle during myogenesis [38]. In cattle, MEF2A is a positive regulator in skeletal muscle myoblast proliferation and differentiation [38, 39] and mutations in its promoter region are highly associated with MEF2A mRNA expression in bulls, which in turn might be related to differences in muscle development and growth traits [40]. As in liver, the enrichment of open chromatin data in human cell lines specific to each tissue confirms the tissue specificity of the peaks/targets, validating our master regulators. 
In cattle, hypothalamus is the least studied tissue of the three, probably due to difficulties to access and correctly identify it. It is also the most complex tissue involved in feedback loops related to the releasing of hormones, regulation of body temperature, maintenance of daily physiological cycles, control of appetite, management of sexual behavior, and regulation of emotional responses [41]. It has three main regions and our open chromatin regions are expected to represent all those regions collectively. It is no surprise then that hypothalamus presented the highest number of peaks and tissuespecific peaks. Indeed, gene expression in hypothalamus when compared to liver and muscle has shown to be higher in indicine cattle [42, 43]. Concordantly with its functions, genes associated with hypothalamus-specific peaks were enriched for terms related to cell communication and nervous system development. Admittedly, our definition of tissue-specific peaks is limited as we are only comparing three tissues and hypothalamus-specific peaks could include open chromatin regions common to other nervous system tissues. Nevertheless, our data points to the SRY-related HMG box (SOX) genes as candidate master regulators of hypothalamus expression.

The HMG box is a DNA-binding domain highly conserved throughout eukaryotic species and the SOX family is divided into subgroups according to homology within this domain and other structural motifs-SOX8, SOX9, and SOX10 are part of Sox group [44]. Apart from SoxE group, SOX3 (SoxB1 group) and SOX6 (SoxD group) also appear in the top 5 enriched transcription factors. Several SOX genes presented expression in hypothalamus and the co-expression of SOX8/9/10 with SOX1 and SOX2 (SoxB1 group); SOX5, SOX6, and SOX13 (SoxD group) and SOX21 (SoxB2 group) show a coordinated activity of this family. SOX genes are related to several different aspects of development and while many are involved in sex determination, some are also important in processes such as neuronal development. Within the tuberal hypothalamus, neural progenitors are known to give rise to supportive and active signaling central nervous system glial cells. This process starts with progenitor cells expressing SOX9 which further mature and start to express SOX10 [45]. As a parallel, in pituitary, Sox $2+/$ Sox9+ cells were demonstrated to be able to generate all hormone-producing cell subtypes [46]. Altogether, SOX genes and in particular group SoxE are indicated as a potential master regulator of hypothalamic gene expression.

Finally, the B. taurus vs B. indicus contrast has long been the subject of studies aimed at characterizing signatures of selection [47]. Mutations affecting complex traits may be subject to natural or artificial selection, which leaves a selection signature in the genome $[48,49]$. However, while the cattle genome has been shaped significantly by human domestication [50], earlier work in cattle suggested few discernible signatures of selection in the cattle genome sequence after strong artificial selection for complex traits [51]. Nevertheless, the epigenome can be responsible for carrying some of the answers for adaptation-related traits that differ across subspecies. In our study, indicinespecific peaks were conspicuously lacking near TSS, which was less apparent for hypothalamus due to the large difference in identified peaks between datasets. For all tissues, most of the peaks were in intergenic regions, corresponding to enhancers, which is in accordance with what was observed when comparing different species $[18,19]$. For instance, a comparison of cattle with pig and mouse showed as little as $17 \%$ and $6 \%$ overlap in intergenic open chromatin, respectively [19]. Enhancers are rapidly evolving regulatory sequences, being a species-specific feature likely to impact differing 
phenotypes [52-55]. In the comparison between the subspecies indicus and taurus, this enhancer-based regulation seems to be even more explicit. Therefore, we trust the indicine-specific open chromatin regions reported here represent a rich source for mining mutations likely to affect cattle adaptation to different climatic zones.

Because most of the indicine-specific peaks were located in intergenic locations, using the nearest gene to draw possible biological functions could be misleading, as enhancers can regulate genes in long distances and even different chromosomes and not necessarily the nearest gene [56]. However, testing the overlap between selective sweeps in Bos indicus and indicine-specific peaks could demonstrate overlapping mechanisms in the control of adaptive differences. Indeed, there was overlap, which mostly happened on chromosome 5. Importantly, hypothalamus peaks on chromosome 5 were distal to HELB, a gene already shown to be related to differences between both cattle subspecies [25].

\section{Conclusions}

A comparative analysis of the chromatin accessibility in muscle, liver, and hypothalamus of Bos indicus cattle revealed new insights into the tissue-specific regulation of gene expression with an unprecedented level of accuracy. The integration of transcriptomic data allowed us to indicate, more accurately, possible targets of master regulators in each tissue, including a prediction of their biding sites. Furthermore, the indication of indicine-specific open chromatin regions provides a promising avenue to exploit molecular mechanisms to artificial selection for traits of relevance to the adaptation to tropical and subtropical climates.

\section{Methods}

\section{Collection of tissue and generation of ATAC-seq libraries}

Liver, hypothalamus, and muscle samples were collected from three unrelated, postpubertal Brahman heifers of similar age and weight as previously described [21-23]. Heifers used in this study were managed, handled, and euthanized as per approval of the Animal Ethics Committee of the University of Queensland, Production and Companion Animal group (certificate number QAAFI/279/12). After slaughter, tissue samples were collected as fast as possible and stored at $-80^{\circ} \mathrm{C}$.

ATAC-seq libraries were prepared from frozen tissues using the Omni-ATAC method [57] with the following modifications. Frozen tissue $(20 \mathrm{mg})$ was ground in liquid nitrogen using a mortar and pestle. The pulverized tissue was transferred to a prechilled $2 \mathrm{ml}$ Dounce homogenizer containing $1 \mathrm{ml}$ cold $1 \times$ homogenization buffer and homogenized with the pestle until a uniform suspension was seen (10-20 strokes). The homogenate was filtered with a $40-\mu \mathrm{M}$ nylon cell strainer (BD Falcon) before layering onto the iodixanol solution as described previously [57]. The ratio of nuclei to enzyme concentration was optimized for each sample by performing transposition reactions containing 50,000, 100,000, and 200,000 nuclei with $2.5 \mu \mathrm{l}$ of tagment enzyme in $50 \mu \mathrm{l}$ of transposition mix [57]. The transposed DNA was amplified with custom primers as previously described [58]. Amplified libraries were purified using Agencourt AMPure XP beads (Beckman Coulter) and quality controlled using a Bioanalyser High Sensitivity DNA Analysis kit (Agilent). ATAC-seq libraries were sequenced at IMB sequencing 
facility (University of Queensland) on an Illumina NextSeq 150 cycle (2X 75 bp). Three biological replicates were performed per tissue. This dataset is publicly available at NCBI Gene Expression Omnibus (GEO) under the accession number GSE182909 [59]. All assays were performed according to FAANG guidelines and recommendations, available at http://www.faang.org. The detailed protocol used in ATAC-seq is available at https://data.faang.org/protocol/samples/ROSLIN_SOP_ATAC_Seq_ DNAIsolationandTagmentation_Frozen_Muscle_Tissue_20200720.pdf.

\section{Mapping and ATAC-seq peak calling}

ATAC-seq data processing and alignment was completed using the Harvard pipeline (https://informatics.fas.harvard.edu/atac-seq-guidelines.html). First, reads quality was accessed using the tool FastQC (https://www.bioinformatics.babraham.ac.uk/projects/ fastqc/). After checking no adapter contamination existed, sample reads were aligned to the cattle reference genome (ARS-UCD1.2) using HISAT2 v2.1.0 with -k 10 to allow for multiple alignments [60]. Summary mapping statistics were performed using Samtools flagstat (v1.9) [61].

Peak calling was performed using the tool Genrich v0.6.1 (available at https://github. com/jsh58/Genrich) including all biological replicates per tissue and parameters $-\mathrm{j}$ (ATAC-seq mode) -r (remove PCR duplicates) -e MT (to exclude mitochondrial chromosome) -p 0.01 ( $p$ value). Genrich analyzes reads that map to multiple locations in the genome by adding a fractional count to each location, allowing for peak detection in regions that are otherwise inaccessible to the assay. Moreover, it calls peaks for multiple biological replicates collectively by first analyzing the replicates separately and then combining the multiple replicates' $p$ values at each genomic position using Fisher's method to identify significant consensus peaks per tissue.

\section{Annotation of peaks and tissue specificity}

After peak calling, peak location was accessed by the R package ChIPseeker [62] using as reference the Bioconductor Bos taurus annotation libraries TxDb.Btaurus.UCSC.bosTau9.refGene [63] and org. Bt.eg.db [64] (A detailed tutorial can be found at https:// www.bioconductor.org/packages/release/bioc/vignettes/ChIPseeker/inst/doc/ ChIPseeker.html). Briefly, the ChIPseeker covplot function was used to calculate and visualize the coverage of peak regions over chromosomes. Then, the profile of peaks binding to TSS regions was visualized by first defining the TSS regions as $\pm 3 \mathrm{~kb}$ of TSS sites, and then aligning the peaks that were mapped to these regions using the ChIPseeker getTagMatrix function and TxDb.Btaurus.UCSC.bosTau9.refGene database as reference. Heatmaps of peak profile around TSS were produced using ChIPseeker tagHeatmap function and peak distribution profiles were produced using ChIPseeker plotAvProf which generate confidence intervals estimated by bootstrap method. Peak annotation to functional categories was performed by ChIPseeker annotatePeak function, which reports the genomic region of the peak (following the priority order: Promoter, 5' UTR, 3' UTR, Exon, Intron, Downstream, and Intergenic), the position and strand of the nearest gene, and the distance to TSS of the nearest gene using the org.Bt.eg.db database as a reference. ChIPseeker plotDistToTSS was used to calculate the 
percentage of peaks upstream and downstream from the TSS of the nearest genes and visualize the distribution.

To compare the three tissues and identify tissue-specific peaks, we used bedtools intersect $-\mathrm{v}$ (v. 2.29.2) [65] for each pairwise contrast. By looking at the results of the multiple contrasts, we defined peaks exclusive to one tissue as tissue-specific. Then, bedtools multiIntersectBed [65] was used to identify overlapping regions across the three tissues. Although a perfect overlap of peaks from different tissues is unlikely, regions of overlap can be of biological significance and will be referred to as constitutive regions. Considering the annotated nearest gene of peaks/regions, we performed an enrichment analysis of Gene Ontology (GO) terms using the function compareCluster from $\mathrm{R}$ package clusterProfiler [66] using the following parameters: OrgDb $=$ org.Bt.eg.db, fun = "enrichGO," ont $=$ "ALL," pAdjustMethod $=$ "BH," pvalueCutoff $=0.05$.

\section{Motif enrichment analysis}

To identify enriched TFBSs within tissue-specific peaks, peaks coordinates in each tissue were converted first to human hg38 coordinates using the liftOver tool [67] (minMatch = 0.1 ), and then to human hg19 coordinates (minMatch $=0.95$ ). The hg19 orthologous regions were used as input to the motif discovery tool i-cisTarget v6.0 [68] which contains 24,453 PWMs gathered from multiple databases (see:http://iregulon.aertslab.org/ collections.html\#motifcolldesc). The i-cisTarget tool contains motif information across seven species, including cow, and those motifs were previously scored for the enrichment of homotypic clusters of PWM using a Hidden Markov Model from the tool ClusterBuster [69]. The seven species whole-genome rankings per motif were combined in a final rank using order statistics to prioritize highly ranked regions per motif across species [70]. The user-defined regions are interrogated for motifs significantly enriched using the cross-species final rank. In addition, it provides the identification of target regions for a PWM by determining the optimal threshold through a receiver operating characteristic curve which compares the enrichment of a PWM versus the enrichment across all 24,453 PWMs average. The Normalized Enrichment Score (NES) is the AUC score normalized by subtracting the mean of all AUC overall motifs and dividing it by the standard deviation. Finally, motifs referring to similar TFs are colour coded and master regulators for each tissue can be determined. For each predicted TF, the top 10 PWMs are gathered to determine which Human regions are predicted as targets.

In addition, i-cisTarget has a collection of 1331 TF ChIP-seq information and 2450 Histone modification tracks in human tissues and cell lines extracted from ENCODE and RoadMap Epigenomics databases [13]. The user-defined regions are interrogated for each track collection and tracks significantly enriched are identified by determining the optimal threshold through a receiver operating characteristic curve. This analysis compares the enrichment of a track of the collection versus the average enrichment across the whole track collection and generates an NES.

\section{Annotating the location of TF binding sites in the cattle genome}

Human (hg19) regions predicted as targets for the top TF in liver, muscle, and hypothalamus were converted back to ARS-UCD1.2 coordinates using the liftOver tool as described before [67]. Next, the overlap between these target regions and ATAC-seq peaks in each 
tissue was calculated using bedtools intersect (-wa -F 0.40) (v2.29.2) [65]. To score and locate the potential TFBSs per TF of interest, we downloaded the PWMs for the top 10 PWMs associated with a TF from the motif discovery analysis [68]. Peaks overlapping target regions for a TF were converted to fasta and re-scanned for the homotypic cluster of PWMs using the Hidden Markov Model from the tool Cluster-Buster (-m 0 -c 0) [69]. The homotypic cluster score, motif score, and predicted binding location were calculated to annotate the active biding sites of the key transcription factor in each tissue.

\section{Gene regulatory network}

To validate the relationships between the master regulator of each tissue (top TF) and its predicted targets at transcriptional level, we used the previously described RNA-seq data of liver and hypothalamus to investigate gene co-expression [21-23]. In addition to the three post-pubertal Brahman heifers used for ATAC-seq libraries, RNA-seq data included three prepubertal Brahman heifers coming from the same original data so the number of samples would enable a co-expression study. This dataset is publicly available at EMBL-EBI BioSamples repository (www.ebi.ac.uk/biosamples) under the submission identifiers GSB-113 and GSB-8708 [71]. RNA-seq reads were aligned to the same cattle reference genome, and read counts were estimated using -tools [72]. The EdgeR R package [73] was used to normalize the counts by TMM (trimmed mean of M values) for each tissue, and only genes presenting at least 1 count per million reads mapped (CPM) in at least half of the samples were considered for further analysis. For each tissue, gene expression in $\log 2 \mathrm{CPM}$ of the master regulator and its predicted targets were used to identify significant connections using the Partial Correlation and Information Theory (PCIT) algorithm [74]. PCIT determinates significant correlations between two genes after accounting for all the other genes under scrutiny.

Gene Regulatory Network visualization was performed using Cytoscape 3.6.0 [75]. Genes having ATAC-seq peaks associated with binding for a TF were drawn in the network as potential target genes and connections validated by co-expression were highlighted.

\section{Identification of Bos indicus-specific open chromatin regions}

To compare open chromatin regions between B. taurus and B. indicus and identify indicine-specific regions, we used data from [19]. Briefly, the authors generated ATAC-seq data from liver, muscle, and hypothalamus of two Hereford males and the identified peaks per sample were available in their Additional File 2. Although there are differences in sex and methods regarding library preparation, sequencing, and peak calling, both studies used the same reference genome which provides us with a unique opportunity to compare results. As described by the authors, peaks called for individual biological replicates were compared with bedtools intersect and then merge collapsed with bedtools merge (v2.29.2) [65]. To identify indicine-specific peaks, we compared indicus and taurus peaks in each tissue using bedtools intersect -v. Peaks were then annotated to functional categories using ChIPseeker annotatePeak [62] as described before. Finally, we compared indicine-specific peaks with regions of selective sweeps for indicine cattle, which were previously identified by our group [25] and are publicly available as Table S5 (selective sweeps in Asian Indicine cattle based on Fst and nucleotide diversity across Bos indicus and Bos taurus cattle, Padj <0.05). For this comparison, we used bedtools intersect -wa to identify overlaps. 


\section{Supplementary Information}

The online version contains supplementary material available at https://doi.org/10.1186/s13059-021-02489-7.

Additional file 1. Summary mapping statistics per sample.

Additional file 2. Identified peaks and annotation for hypothalamus. The first 10 columns correspond to ENCODE narrowPeak format, the following columns are the annotation output of ChIPseeker [62], and the last column indicates which of the peaks are tissue-specific.

Additional file 3. Identified peaks and annotation for liver. The first 10 columns correspond to ENCODE narrowPeak format, the following columns are the annotation output of ChIPseeker [62], and the last column indicates which of the peaks are tissue-specific.

Additional file 4. Identified peaks and annotation for muscle. The first 10 columns correspond to ENCODE narrowPeak format, the following columns are the annotation output of ChIPseeker [62], and the last column indicates which of the peaks are tissue-specific.

Additional file 5. Distribution of peaks by chromosome for muscle (A), liver (B) and hypothalamus (C).

Additional file 6. Complete distribution of genomic features overlapping peaks identified in muscle (A), liver (B) and hypothalamus (C).

Additional file 7. Identified peaks and annotation for constitutive regions. The first 10 columns correspond to ENCODE narrowPeak format and the following columns are the annotation output of ChIPseeker [62].

Additional file 8. Functional enrichment of gene ontology terms for tissue-specific peaks.

Additional file 9. (AdditionalFile9.pdf) - Enriched regulatory features in liver-specific peaks according to i-cisTarget online tool [68].

Additional file 10. Annotation of possible binding sites of NHF4 in cattle liver according to Cluster-Buster [69].

Additional file 11. Significant partial correlation between HNF4 and its possible targets in liver using RNAseq data.

Additional file 12. Enriched regulatory features in muscle-specific peaks according to i-cisTarget online tool [68].

Additional file 13. Annotation of possible binding sites of MEF2 in cattle muscle according to Cluster-Buster [69].

Additional file 14. Enriched regulatory features in hypothalamus-specific peaks according to i-cisTarget online tool [68].

Additional file 15. Annotation of possible binding sites of SOX8/9/10 in cattle hypothalamus according to Cluster-Buster [69].

Additional file 16. Hypothalamus-specific master regulator SOX and its predicted targets. Dotted edges represent predicted targets, continuous edges and red borders represent targets with significant co-expression using RNAseq data.

Additional file 17. Significant partial correlation between $50 X 8 / 9 / 10$ and its possible targets in hypothalamus using RNAseq data.

Additional file 18. Indicine-specific peaks in liver, muscle, and hypothalamus.

Additional file 19. Indicine-specific peaks in liver, muscle, and hypothalamus which overlap selective sweeps in indicine cattle.

Additional file 20. Peer review history.

\section{Acknowledgements}

We thank Dr Kerry Roper for her advice on ATAC-seq profiling and lending us some reagents and Dr David Gorkin for his advice on ATAC-seq libraries quality control. We also thank Dr Stephen S. Moore for coordinating the project that generated the RNA-seq used here as part of a FAANG initiative. The authors acknowledge the financial support provided by Meat and Livestock Australia (MLA project L.GEN.1710).

\section{Review history}

The review history is available as Additional file 20.

\section{Peer review information}

Tim Sands was the primary editor of this article and managed its editorial process and peer review in collaboration with the rest of the editorial team.

\section{Authors' contributions}

A.R. and M.N.S. conceived the study. M.M. performed the ATAC-seq libraries. M.N.S., P.A.A, and L.T.N. performed the computational analysis. L.P.N and M.F.R. collected and gave access to the tissue samples. P.A.A., A.R., and M.N.S. wrote the manuscript. The authors read and approved the final manuscript.

\section{Funding}

M.N.S. and P.A.A. were funded by the CSIRO Science Excellence Research Office.

\section{Availability of data and materials}

The ATAC-seq dataset supporting the conclusions of this article is publicly available at NCBI Gene Expression Omnibus (https:/www.ncbi.nlm.nih.gov/geo/) under the accession number GSE182909 [59]. The RNA-seq dataset used to build 
the co-expression networks is publicly available at EMBL-EBI BioSamples repository (www.ebi.ac.uk/biosamples) under the submission identifiers GSB-113 and GSB-8708 [71].

\section{Declarations}

\section{Ethics approval and consent to participate}

Heifers used in this study were managed, handled, and euthanized as per approval of the Animal Ethics Committee of the University of Queensland, Production and Companion Animal group, certificate number QAAFI/279/12.

\section{Consent for publication}

Not applicable

\section{Competing interests}

The authors declare they have no competing interests.

\section{Author details}

${ }^{1}$ CSIRO Agriculture \& Food, 306 Carmody Rd., QLD 4067 Brisbane, Australia. ${ }^{2}$ Institute for Molecular Bioscience, The University of Queensland, Brisbane, QLD 4072, Australia. ${ }^{3}$ Queensland Alliance for Agriculture and Food Innovation, The University of Queensland, Brisbane, QLD 4072, Australia. ${ }^{4}$ School of Chemistry and Molecular Biosciences, The University of Queensland, Brisbane, QLD 4072, Australia.

\section{Received: 21 December 2020 Accepted: 8 September 2021}

Published online: 21 September 2021

\section{References}

1. Yan F, Powell DR, Curtis DJ, Wong NC. From reads to insight: a hitchhiker's guide to ATAC-seq data analysis. Genome Biol. 2020;21:22. Available from: https://genomebiology.biomedcentral.com/articles/10.1186/s13059-020-1929-3

2. Radman-Livaja M, Rando OJ. Nucleosome positioning: how is it established, and why does it matter? Dev Biol. 2010; 339(2):258-66. Available from: http://10.0.3.248/i.ydbio.2009.06.012. https://doi.org/10.1016/j.ydbio.2009.06.012.

3. Klemm SL, Shipony Z, Greenleaf WJ. Chromatin accessibility and the regulatory epigenome. Nat Rev Genet; 2019;20: 207-220. Available from: http://10.0.4.14/s41576-018-0089-8, DOl: https://doi.org/10.1038/s41576-018-0089-8

4. Moore JE, Purcaro MJ, Pratt HE, Epstein CB, Shoresh N, Adrian J, et al. Expanded encyclopaedias of DNA elements in the human and mouse genomes. Nature. 2020;583:699-710. Available from: http://www.nature.com/articles/s41586-020-24 93-4

5. ENCODE Project Consortium, ENCODE Project Consortium T, Coordination O, production leads D, Analysts L, Group W, et al. An integrated encyclopedia of DNA elements in the human genome. Nature. 2012;489:57-74. Available from: http://encodeproject.org/ENCODE/

6. Yue F, Cheng Y, Breschi A, Vierstra J, Wu W, Ryba T, et al. A comparative encyclopedia of DNA elements in the mouse genome. Nature. 2014;515:355-64 Available from: http://10.0.4.14/nature13992.

7. Gerstein MB, Lu ZJ, Van Nostrand EL, Cheng C, Arshinoff BI, Liu T, et al. Integrative Analysis of the Caenorhabditis elegans Genome by the modENCODE Project. Science (80- ). 2010;330:1775-87. Available from: https://www.sciencema g.org/lookup/doi/10.1126/science.1196914

8. Roy S, Ernst J, Kharchenko P V., Kheradpour P, Negre N, Eaton ML, et al. Identification of functional elements and regulatory circuits by Drosophila modENCODE. Science (80- ). 2010;330:1787-97. Available from: https://www.sciencema g.org/lookup/doi/10.1126/science.1198374

9. Giuffra E, Tuggle CK. Functional Annotation of Animal Genomes (FAANG): Current Achievements and Roadmap. Annu Rev Anim Biosci. 2019;7(1):65-88. Available from: https://www.annualreviews.org/doi/10.1146/annurev-animal-02051 8-114913.

10. Macqueen DJ, Primmer CR, Houston RD, Nowak BF, Bernatchez L, Bergseth S, et al. Functional Annotation of All Salmonid Genomes (FAASG): an international initiative supporting future salmonid research, conservation and aquaculture. BMC Genomics. 2017;18:484. Available from: https://bmcgenomics.biomedcentral.com/articles/10.1186/s12 864-017-3862-8

11. Naval-Sanchez M, Nguyen Q, McWilliam S, Porto-Neto LR, Tellam R, Vuocolo T, Reverter A, Perez-Enciso M, Brauning R, Clarke S, McCulloch A, Zamani W, Naderi S, Rezaei HR, Pompanon F, Taberlet P, Worley KC, Gibbs RA, Muzny DM, Jhangiani SN, Cockett N, Daetwyler H, Kijas I Sheep genome functional annotation reveals proximal regulatory elements contributed to the evolution of modern breeds. Nat Commun; 2018;9:859. Available from: http://10.0.4.14/s41467-01702809-1, DOl: https://doi.org/10.1038/s41467-017-02809-1

12. Nguyen QH, Tellam RL, Naval-Sanchez M, Porto-Neto LR, Barendse W, Reverter A, et al. Mammalian genomic regulatory regions predicted by utilizing human genomics, transcriptomics, and epigenetics data. Gigascience. Oxford University Press (OUP); 2018;7. Available from: http://10.0.4.69/gigascience/gix136

13. Kundaje A, Meuleman W, Ernst J, Bilenky M, Yen A, Heravi-Moussavi A, et al. Integrative analysis of 111 reference human epigenomes. Nature. 2015;518:317-30 Available from: http://www.nature.com/articles/nature14248.

14. Porto-Neto LR, Sonstegard TS, Liu GE, Bickhart DM, Da Silva MVB, Machado MA, et al. Genomic divergence of zebu and taurine cattle identified through high-density SNP genotyping. BMC Genomics. 2013;14:876. Available from: http:// bmcgenomics.biomedcentral.com/articles/10.1186/1471-2164-14-876

15. Naval-Sánchez M, Porto-Neto LR, Cardoso DF, Hayes BJ, Daetwyler HD, Kijas J, et al. Selection signatures in tropical cattle are enriched for promoter and coding regions and reveal missense mutations in the damage response gene HELB. Genet Sel Evol; 2020;52:27. Available from: http://10.0.4.162/s12711-020-00546-6

16. Robinson TP, Wint GRW, Conchedda G, Van Boeckel TP, Ercoli V, Palamara E, et al. Mapping the Global Distribution of Livestock. Baylis M, editor. PLoS One. 2014;9:e96084. Available from: https://dx.plos.org/10.1371/journal.pone.0096084 
17. Tsompana M, Buck MJ. Chromatin accessibility: a window into the genome. Epigenetics Chromatin.; 2014;7:33. Available from: http://10.0.4.162/1756-8935-7-33

18. Foissac S, Djebali S, Munyard K, Vialaneix N, Rau A, Muret K, et al. Multi-species annotation of transcriptome and chromatin structure in domesticated animals. BMC Biol. 2019;17:108. Available from: https://bmcbiol.biomedcentral. com/articles/10.1186/s12915-019-0726-5

19. Halstead MM, Kern C, Saelao P, Wang Y, Chanthavixay G, Medrano JF, et al. A comparative analysis of chromatin accessibility in cattle, pig, and mouse tissues. BMC Genomics. 2020;21 (1):698. Available from: https://bmcgenomics. biomedcentral.com/articles/10.1186/s12864-020-07078-9.

20. McGavin MD, Zachary JJ. Pathologic Basis of Veterinary Disease. 4th ed. Elsevier; 2007.

21. Fortes MRS, Nguyen LT, Weller MMDCA, Cánovas A, Islas-Trejo A, Porto-Neto LR, et al. Transcriptome analyses identify five transcription factors differentially expressed in the hypothalamus of post- versus prepubertal Brahman heifers. J Anim Sci. 2016;94:3693-702. Available from: http://www.ncbi.nlm.nih.gov/pubmed/27898892, 2016

22. Nguyen LT, Reverter A, Cánovas A, Venus B, Anderson ST, Islas-Trejo A, et al. STAT6, PBX2, and PBRM1 emerge as predicted regulators of 452 differentially expressed genes associated with puberty in Brahman heifers. Front Genet. 2018;9:87. Available from: http://www.ncbin.nlm.nih.gov/pubmed/29616079, 2018

23. Lau LY, Nguyen LT, Reverter A, Moore SS, Lynn A, McBride-Kelly L, et al. Gene regulation could be attributed to TCF3 and other key transcription factors in the muscle of pubertal heifers. Vet Med Sci. 2020;6:695-710. Available from: https://onlinelibrary.wiley.com/doi/10.1002/vms3.278

24. ENCODE Project Consortium. ATAC-seq data standards and processing pipeline. Available from: https://www. encodeproject.org/atac-seq/

25. Naval-Sánchez M, Porto-Neto LR, Cardoso DF, Hayes BJ, Daetwyler HD, Kijas J, et al. Selection signatures in tropical cattle are enriched for promoter and coding regions and reveal missense mutations in the damage response gene HELB. Genet Sel Evol. 2020;52:27. Available from: https://gsejournal.biomedcentral.com/articles/1 0.1186/s12711-020-00546-6

26. Schoenfelder S, Fraser P. Long-range enhancer-promoter contacts in gene expression control. Nat Rev Genet:; 2019;20: 437-455. Available from: https://doi.org/10.1038/s41576-019-0128-0, 8

27. Yao L, Berman BP, Farnham PJ. Demystifying the secret mission of enhancers: Linking distal regulatory elements to target genes. Crit Rev Biochem Mol Biol. 2015;50(6):550-73. https://doi.org/10.3109/10409238.2015.1087961.

28. Bruce AW, Donaldson IJ, Wood IC, Yerbury S a, Sadowski MI, Chapman M, et al. Enhancer function: new insights into the regulation of tissue- specific gene expression. Nature. 2011;473:10458-63. Available from: http://www.ncbi.nIm.nih. gov/pubmed/21593866\%5Cn; http://www.pubmedcentral.nih.gov/articlerender.fcgi?artid=478591\&tool= pmcentrez\&rendertype=abstract

29. Lorberbaum DS, Barolo S. Enhancers: holding out for the right promoter. Curr Biol. 2015;25(7):R290-3. Available from: https://inkinghub.elsevier.com/retrieve/pii/S0960982215000718. https://doi.org/10.1016/j.cub.2015.01.039.

30. Rappel W, Loomis WF. Eukaryotic chemotaxis. Wiley Interdiscip Rev Syst Biol Med. 2009;1 (1):141-9. Available from: https://www.ncbi.nlm.nih.gov/pmc/articles/PMC3624763/pdf/nihms412728.pdf. https://doi.org/10.1002/wsbm.28.

31. Kubes P, Jenne C. Immune responses in the liver. Annu Rev Immunol. 2018;36(1):247-77. Available from: http://www.a nnualreviews.org/doi/10.1146/annurev-immunol-051116-052415.

32. Robinson MW, Harmon C, O'Farrelly C. Liver immunology and its role in inflammation and homeostasis. Cell Mol Immunol. 2016;13(3):267-76. https://doi.org/10.1038/cmi.2016.3.

33. Lau HH, Ng NHJ, Loo LSW, Jasmen JB, Teo AKK. The molecular functions of hepatocyte nuclear factors - In and beyond the liver. J Hepatol. 2018;68(5):1033-48. Available from: https://linkinghub.elsevier.com/retrieve/pii/S0168827817324510. https://doi.org/10.1016/j.jhep.2017.11.026.

34. Costa RH, Kalinichenko W, Holterman AXL, Wang X. Transcription factors in liver development, differentiation, and regeneration. Hepatology. 2003;38(6):1331-47. https://doi.org/10.1016/j.hep.2003.09.034.

35. Drewes T, Senkel S, Holewa B, Ryffel GU. Human hepatocyte nuclear factor 4 isoforms are encoded by distinct and differentially expressed genes. Mol Cell Biol. 1996;16:925-31. Available from: https://journals.asm.org/doi/10.1128/MCB.1 6.3 .925

36. Ramayo-Caldas Y, Fortes MRS, Hudson NJ, Porto-Neto LR, Bolormaa S, Barendse W, et al. A marker-derived gene network reveals the regulatory role of PPARGC1A, HNF4G, and FOXP3 in intramuscular fat deposition of beef cattle1. J Anim Sci. 2014;92:2832-45. Available from: https:/academic.oup.com/jas/article/92/7/2832/4702130

37. Pon JR, Marra MA. MEF2 transcription factors: developmental regulators and emerging cancer genes. Oncotarget. 2016; 7(3):2297-312. Available from: https://www.oncotarget.com/lookup/doi/10.18632/oncotarget.6223.

38. Wang Y-N, Yang W-C, Li P-W, Wang H-B, Zhang Y-Y, Zan L-S. Myocyte enhancer factor 2A promotes proliferation and its inhibition attenuates myogenic differentiation via myozenin 2 in bovine skeletal muscle myoblast. te Pas MFW, editor. PLoS One. 2018;13:e0196255. Available from: https://dx.plos.org/10.1371/journal.pone.0196255

39. Wang Y, Mei C, Su X, Wang H, Yang W, Zan L. MEF2A regulates the MEG3-DIO3 miRNA mega cluster-targeted PP2A signaling in bovine skeletal myoblast differentiation. Int J Mol Sci. 2019;20:2748. Available from: https://www.mdpi. com/1422-0067/20/11/2748

40. Juszczuk-Kubiak E, Starzyński RR, Wicińska K, Flisikowski K. Promoter variant-dependent mRNA expression of the MEF2A in longissimus dorsi muscle in cattle. DNA Cell Biol. 2012;31(6):1131-5. https://doi.org/10.1089/dna.2011.1533.

41. Cunningham JG, Klein BG. Veterinary Physiology. Fourth. Duncan L, editor. St. Louis, Missouri: Elsevier Ltd; 2007.

42. Alexandre PA, Naval-Sanchez M, Porto-Neto LR, Ferraz JBS, Reverter A, Fukumasu H. Systems biology reveals NR2F6 and TGFB1 as key regulators of feed efficiency in beef cattle. Front Genet. 2019;10:1-16. Available from: http://biorxiv.org/ content/early/2018/07/02/360396.abstract

43. Alexandre PA, Reverter A, Berezin RB, Porto-Neto LR, Ribeiro G, Santana MHA, et al. Exploring the regulatory potential of long non-coding RNA in feed efficiency of indicine cattle. Genes (Basel). 2020;11:997. Available from: https://www.mdpi. com/2073-4425/11/9/997

44. Bowles J, Schepers G, Koopman P. Phylogeny of the SOX family of developmental transcription factors based on sequence and structural indicators. Dev Biol. 2000;227:239-55. Available from: https://inkinghub.elsevier.com/retrieve/ pii/S001216060099883X 
45. Marsters CM, Rosin JM, Thornton HF, Aslanpour S, Klenin N, Wilkinson G, et al. Oligodendrocyte development in the embryonic tuberal hypothalamus and the influence of Ascl1. Neural Dev. 2016;11:20. Available from: http://neura Idevelopment.biomedcentral.com/articles/10.1186/s13064-016-0075-9

46. Rizzoti K, Akiyama H, Lovell-Badge R. Mobilized adult pituitary stem cells contribute to endocrine regeneration in response to physiological demand. Cell Stem Cell. 2013;13:419-32. Available from: https://linkinghub.elsevier.com/ retrieve/pii/S1934590913003147

47. Randhawa IAS, Khatkar MS, Thomson PC, Raadsma HW. A meta-assembly of selection signatures in cattle. Barendse W, editor. PLoS One. 2016;11:e0153013. Available from: https://dx.plos.org/10.1371/journal.pone.0153013

48. Zeng J, de Vlaming R, Wu Y, Robinson MR, Lloyd-Jones LR, Yengo L, et al. Signatures of negative selection in the genetic architecture of human complex traits. Nat Genet. 2018;50(5):746-53. Available from: http://www.nature.com/a rticles/s41588-018-0101-4. https://doi.org/10.1038/s41588-018-0101-4.

49. Yang J, Jin Z-B, Chen J, Huang X-F, Li X-M, Liang Y-B, et al. Genetic signatures of high-altitude adaptation in Tibetans. Proc Natl Acad Sci. 2017;114(16):4189-94. Available from: http://www.pnas.org/lookup/doi/10.1073/pnas.1617042114.

50. Xu L, Bickhart DM, Cole JB, Schroeder SG, Song J, Van Tassell CP, et al. Genomic signatures reveal new evidences for selection of important traits in domestic cattle. Mol Biol Evol. 2015;32(3):711-25. Available from: https://academic.oup. com/mbe/article-lookup/doi/10.1093/molbev/msu333.

51. Kemper KE, Saxton SJ, Bolormaa S, Hayes BJ, Goddard ME. Selection for complex traits leaves little or no classic signatures of selection. BMC Genomics. 2014;15:246. Available from: http://bmcgenomics.biomedcentral.com/articles/1 $0.1186 / 1471-2164-15-246$

52. Shibata Y, Sheffield NC, Fedrigo O, Babbitt CC, Wortham M, Tewari AK, et al. Extensive evolutionary changes in regulatory element activity during human origins are associated with altered gene expression and positive selection. Akey JM, editor. PLoS Genet. 2012;8:e1002789. Available from: https://dx.plos.org/10.1371/journal.pgen.1002789

53. Xiao S, Xie D, Cao X, Yu P, Xing X, Chen C-C, et al. Comparative epigenomic annotation of regulatory DNA. Cell. 2012; 149(6):1381-92. Available from: https://linkinghub.elsevier.com/retrieve/pii/S0092867412005740. https://doi.org/10.1016/j. cell.2012.04.029.

54. Yue F, Cheng Y, Breschi A, Vierstra J, Wu W, Ryba T, et al. A comparative encyclopedia of DNA elements in the mouse genome. Nature. 2014;515(7527):355-64. Available from: http://www.nature.com/articles/nature13992. https://doi.org/1 0.1038 /nature13992.

55. Villar D, Berthelot C, Aldridge S, Rayner TF, Lukk M, Pignatelli M, et al. Enhancer evolution across 20 mammalian species. Cell. 2015;160(3):554-66. https://doi.org/10.1016/j.cell.2015.01.006.

56. Alberts B, Johnson A, Lewis J, Raff M, Roberts K, Walter P. Molecular Biology of Cell. 5th ed; 2008. https://doi.org/10.12 01/9780203833445.

57. Corces MR, Trevino AE, Hamilton EG, Greenside PG, Sinnott-Armstrong NA, Vesuna S, et al. An improved ATAC-seq protocol reduces background and enables interrogation of frozen tissues. Nat Methods. 2017;14:959-62. Available from: http://www.nature.com/articles/nmeth.4396

58. Buenrostro JD, Wu B, Chang HY, Greenleaf WJ. ATAC-seq: a method for assaying chromatin accessibility genome-wide. Curr Protoc Mol Biol. Hoboken, NJ, USA: John Wiley \& Sons, Inc.; 2015;109:21.29.1-21.29.9. Available from: https:// onlinelibrary.wiley.com/doi/abs/10.1002/0471142727.mb2129s109

59. Alexandre P, Naval-Sanchez M, Menzies M, Nguyen L, Porto-Neto L, MR F, et al. Chromatin accessibility and regulatory vocabulary across indicine cattle tissues. GSE182909. NCBI GEO. 2021. Available from: https://www.ncbi.nlm.nih.gov/geo/ query/acc.cgi?acc=GSE182909

60. Kim D, Langmead B, Salzberg SL. HISAT: a fast spliced aligner with low memory requirements. Nat Methods. 2015;12: 357-60. Available from: http://www.ncbi.nlm.nih.gov/pubmed/25751142, 2015

61. Li H, Handsaker B, Wysoker A, Fennell T, Ruan J, Homer N, et al. The Sequence Alignment/Map format and SAMtools. Bioinformatics. 2009;25:2078-9. Available from: http://www.pubmedcentral.nih.gov/articlerender.fcgi?artid=2723002 \&tool=pmcentrez\&rendertype=abstract

62. Yu G, Wang L-G, He Q-Y. ChIPseeker: an R/Bioconductor package for ChIP peak annotation, comparison and visualization. Bioinformatics. 2015;31(14):2382-3. Available from: https://academic.oup.com/bioinformatics/article-lookup/ doi/10.1093/bioinformatics/btv145.

63. Bioconductor Core Team and Bioconductor Package Maintainer. TxDb.Btaurus.UCSC.bosTau9.refGene: Annotation package for TxDb object(s). Bioconductor Package Maintainer; 2019. Available from: http://www.bioconductor.org/packa ges/release/data/annotation/html/TxDb.Btaurus.UCSC.bosTau9.refGene.html

64. Carlson M. org.Bt.eg.db: Genome wide annotation for Bovine. Bioconductor Package Maintainer; 2019. Available from: https://bioconductor.org/packages/release/data/annotation/html/org.Bt.eg.db.html

65. Quinlan AR, Hall IM. BEDTools: a flexible suite of utilities for comparing genomic features. Bioinformatics. 2010;26(6):8412. Available from: https://academic.oup.com/bioinformatics/article-lookup/doi/10.1093/bioinformatics/btq033.

66. $Y u$ G, Wang L-G, Han Y, He Q-Y. clusterProfiler: an R package for comparing biological themes among gene clusters. Omi A J Integr Biol. 2012;16:284-7. Available from: http://www.liebertpub.com/doi/10.1089/omi.2011.0118

67. Kent WJ, Zweig AS, Barber G, Hinrichs AS, Karolchik D. BigWig and BigBed: enabling browsing of large distributed datasets. Bioinformatics. 2010;26:2204-7. Available from: https://academic.oup.com/bioinformatics/article-lookup/doi/10.1 093/bioinformatics/btq351

68. Imrichová H, Hulselmans G, Kalender Atak Z, Potier D, Aerts S. i-cisTarget 2015 update: generalized cis-regulatory enrichment analysis in human, mouse and fly. Nucleic Acids Res. 2015;43:W57-64. Available from: https://academic.oup. com/nar/article-lookup/doi/10.1093/nar/gkv395

69. Frith MC. Cluster-Buster: finding dense clusters of motifs in DNA sequences. Nucleic Acids Res. 2003;31(13):3666-8. Available from: https://academic.oup.com/nar/article-lookup/doi/10.1093/nar/gkg540.

70. Aerts S, Lambrechts D, Maity S, Van Loo P, Coessens B, De Smet F, et al. Gene prioritization through genomic data fusion. Nat Biotechnol. 2006;24(5):537-44. Available from: http://www.nature.com/articles/nbt1203. https://doi.org/10.1038/nbt1203.

71. Fortes MRS, Nguyen LT, Weller MMDCA, Cánovas A, Islas-Trejo A, Porto-Neto LR, et al. Total and small RNA of testicular tissues (foetal and adult) and liver (adult) from Bos indicus cattle. GSB-113, GSB-8708. EMBL-EBI. 2018. Available from: https://www.ebi.ac.uk/biosamples/samples/SAMEG100056 
72. Anders S, Pyl PT, Huber W. HTSeq--a Python framework to work with high-throughput sequencing data. Bioinformatics. 2015;31:166-9. Available from: http://www.pubmedcentral.nih.gov/articlerender.fcgi?artid=4287950\&tool= pmcentrez\&rendertype $=$ abstract

73. Robinson MD, McCarthy DJ, Smyth GK. edgeR: a Bioconductor package for differential expression analysis of digital gene expression data. Bioinformatics. 2010;26:139-40. Available from: http://www.pubmedcentral.nih.gov/articlerender. fcgi?artid=2796818\&tool=pmcentrez\&rendertype $=$ abstract

74. Reverter A, Chan EKF. Combining partial correlation and an information theory approach to the reversed engineering of gene co-expression networks. Bioinformatics. 2008;24:2491-7. Available from: http://www.ncbi.nlm.nih.gov/pubmed/1 8784117

75. Shannon P, Markiel A, Ozier O, Baliga NS, Wang JT, Ramage D, et al. Cytoscape: a software environment for integrated models of biomolecular interaction networks. Genome Res. 2003;13:2498-504. Available from: http://www. pubmedcentral.nih.gov/articlerender.fcgi?artid=403769\&tool=pmcentrez\&rendertype=abstract

\section{Publisher's Note}

Springer Nature remains neutral with regard to jurisdictional claims in published maps and institutional affiliations.

- fast, convenient online submission

- thorough peer review by experienced researchers in your field

- rapid publication on acceptance

- support for research data, including large and complex data types

- gold Open Access which fosters wider collaboration and increased citations

- maximum visibility for your research: over $100 \mathrm{M}$ website views per year

At BMC, research is always in progress. 